\title{
openheart Discordant severity criteria in patients with moderate aortic stenosis: prognostic implications
}

Stephan M Pio, ${ }^{1}$ Mohammed R Amanullah, ${ }^{2}$ Steele C Butcher,${ }^{1,3}$ Kenny Y Sin, ${ }^{4}$
Nina Ajmone Marsan, ${ }^{1}$ Philippe Pibarot (D) ${ }^{5}$ Nicolas M Van Mieghem, ${ }^{6}$ Zee Pin Ding, ${ }^{2}$ Philippe Généreux, ${ }^{7}$ Martin B Leon, ${ }^{8}$ See Hooi Ewe, ${ }^{2}$ Victoria Delgado (D) , Jeroen J Bax ${ }^{1}$

To cite: Pio SM, Amanullah MR, Butcher SC, et al. Discordant severity criteria in patients with moderate aortic stenosis: prognostic implications. Open Heart 2021;8:e001639. doi:10.1136/ openhrt-2021-001639

SMP and MRA contributed equally.

Received 2 March 2021 Accepted 4 June 2021

Check for updates

\section{(c) Author(s) (or their} employer(s)) 2021. Re-use permitted under CC BY-NC. No commercial re-use. See rights and permissions. Published by BMJ.

For numbered affiliations see end of article.

Correspondence to Dr Jeroen J Bax; j.j.bax@lumc.nl

\section{ABSTRACT}

Background The criteria to define the grade of aortic stenosis (AS) - aortic valve area (AVA) and mean gradient (MG) or peak jet velocity — do not always coincide into one grade. Although in severe AS, this discrepancy is well characterised, in moderate AS, the phenomenon of discordant grading has not been investigated and its prognostic implications are unknown.

Objectives To investigate the occurrence of discordant grading in patients with moderate AS (defined by an AVA between $1.0 \mathrm{~cm}^{2}$ and $1.5 \mathrm{~cm}^{2}$ but with an $M G<20 \mathrm{~mm}$ $\mathrm{Hg}$ ) and how these patients compare with those with concordant grading moderate AS (AVA between $1.0 \mathrm{~cm}^{2}$ and $1.5 \mathrm{~cm}^{2}$ and $M G \geq 20 \mathrm{~mm} \mathrm{Hg}$ ) in terms of clinical outcomes.

Methods From an ongoing registry of patients with AS, patients with moderate AS based on AVA were selected and classified into discordant or concordant grading (MG $<20 \mathrm{~mm} \mathrm{Hg}$ or $\geq 20 \mathrm{~mm} \mathrm{Hg}$, respectively). The clinical endpoint was all-cause mortality.

Results Of 790 patients with moderate AS, 150 (19.0\%) had discordant grading, moderate AS. Patients with discordant grading were older, had higher prevalence of previous myocardial infarction and left ventricular (LV) hypertrophy, larger LV end-diastolic and end-systolic volume index, higher LV filling pressure and lower LV ejection fraction and stroke volume index as compared with their counterparts. After a median follow-up of 4.9 years (IQR 3.0-8.2), patients with discordant grading had lower aortic valve replacement rates $(26.7 \%$ vs $44.1 \%$, $p<0.001)$ and higher mortality rates $(60.0 \%$ vs $43.1 \%$, $\mathrm{p}<0.001$ ) as compared with patients with concordant grading. Discordant grading moderate AS, combined with low LV ejection fraction, presented the higher risk of mortality (HR 2.78 (2.00-3.87), p<0.001).

Conclusion Discordant-grading moderate AS is not uncommon and, when combined with low LV ejection fraction, is associated with high risk of mortality.

\section{INTRODUCTION}

Grading aortic stenosis (AS) with echocardiography requires accurate assessment of aortic valve morphology and haemodynamics including the measurement of

\section{Key questions}

What is already known about this subject?

- Patients with moderate aortic stenosis (AS) have worse prognosis compared with general population and patients with mild AS.

- Misclassification of AS severity may lead to longer lasting burden of increased afterload to the left heart.

What does this study add?

- Prevalence of discordant severity criteria in patients with moderate AS and predominantly preserved left ventricular ejection fraction (LVEF) is high.

- Discordant grading is associated with poor prognosis, particularly among patients with LVEF $<50 \%$.

How might this impact on clinical practice?

- Patients with moderate AS and discordant gradient might benefit from a closer follow-up and multimodality imaging.

- The discrepancy between aortic valve area and mean gradient is to be confirmed in TAVR-UNLOAD trial with a probably higher prevalence due to LV systolic dysfunction.

the peak jet velocity and the calculation of the mean transvalvular gradient and aortic valve area (AVA). Among patients with severe AS, one-third can have discordant criteria (AVA $<1.0 \mathrm{~cm}^{2}$ with low transvalvular gradient $(<40 \mathrm{~mm} \mathrm{Hg})$ or peak jet velocity $(<4 \mathrm{~min} / \mathrm{s}))$ posing a diagnostic and therapeutic challenge. ${ }^{1}$ Low flow status, inaccurate measurement of the left ventricular (LV) outflow tract cross-sectional area and misalignment of the aortic jet with the ultrasound beam are frequent reasons that lead to discordant grading. While in severe AS, the occurrence and clinical implications of discordant grading have been extensively investigated, ${ }^{2-7}$ in patients with moderate AS, these questions have not been evaluated. Moderate AS has been associated with 
impaired survival at follow-up. ${ }^{8}$ The ongoing TAVRUNLOAD (Transcatheter Aortic Valve Replacement to UNload the Left Ventricle in Patients With ADvanced Heart Failure) trial is currently recruiting patients with symptomatic heart failure and moderate AS who are randomised to conventional treatment (guidelinebased medical therapy and valve intervention when AS becomes severe) versus transcatheter aortic valve implantation. ${ }^{10}$

The prevalence of discordant grading among patients with moderate AS (overall and according to LV ejection fraction), and its prognostic implications have not been evaluated. Accordingly, the present retrospective multicentre study aimed at evaluating the prevalence of discordant-grading moderate AS and investigating its prognostic implications.

\section{METHODS \\ Patient population}

From the echocardiographic database of two tertiary centres (Leiden University Medical Center, Leiden, The Netherlands and the National Heart Centre Singapore, Singapore), patients who were diagnosed with moderate AS with AVA between $1.0 \mathrm{~cm}^{2}$ and $1.5 \mathrm{~cm}^{2}$ were selected. Patients with moderate or severe coexisting aortic regurgitation, dynamic subaortic obstruction, unavailable echocardiographic data allowing offline two-dimensional analysis and active endocarditis were excluded. Patients included in this analysis were further dichotomised according to concordant mean gradient (MG) and AVA for moderate AS (MG $\geq 20 \mathrm{~mm} \mathrm{Hg}$ and AVA between $1.0 \mathrm{~cm}^{2}$ and $\left.1.5 \mathrm{~cm}^{2}\right)$ versus discordant grading $\left(\mathrm{MG}<20 \mathrm{~mm} \mathrm{Hg}\right.$ and AVA between $1.0 \mathrm{~cm}^{2}$ and $1.5 \mathrm{~cm}^{2}$ ). Demographic and clinical data (cardiovascular risk factors and medication use) as well as clinical outcomes (all-cause mortality) were collected using the hospital records and departmental patient information systems and analysed retrospectively.

This retrospective analysis of clinically acquired data was approved by the respective institutional review boards of each participating centre, and the need for patient written informed consent was waived due to the retrospective nature of the study.

\section{Echocardiography}

Transthoracic echocardiography was performed with the subjects at rest using commercially available ultrasound systems. All images were digitally stored on hard disks for offline analysis with proprietary software. A complete two-dimensional, colour, pulsed and continuous-wave Doppler echocardiogram was performed. LV end-diastolic volume and end-systolic volume were calculated using Simpson's biplane method of discs and corrected for body surface area. left ventricular ejection fraction (LVEF) was calculated and expressed as a percentage. LV mass index was calculated from the formula as recommended by the
American Society of Echocardiography and the European Association of Cardiovascular Imaging. ${ }^{11}$ The cutoff values of $95 \mathrm{~g} / \mathrm{m}^{2}$ for women and $115 \mathrm{~g} / \mathrm{m}^{2}$ for men were used to define LV hypertrophy.

Mitral inflow velocities were recorded using conventional pulsed-wave Doppler echocardiography in the apical four-chamber view using a $2 \mathrm{~mm}$ sample volume. Transmitral early (E wave) and late (A wave) diastolic velocities as well as deceleration time were recorded at the mitral leaflet tips. Tissue Doppler imaging in the apical four-chamber view with measurement of the peak velocities in early diastole of the septal (e' septal) and lateral (e' lateral) basal regions were obtained,and the $\mathrm{LV}$ filling pressures were estimated using the $\mathrm{E} / \mathrm{e}^{\prime}$ ratio.

On a zoomed parasternal long-axis view, the LV outflow tract (LVOT) diameter was measured and the cross-sectional area was derived. From the apical LV long-axis or five-chamber views, continuous wave and pulsed wave Doppler spectral recordings were obtained through the aortic valve and at the LVOT, respectively. The peak and mean aortic pressure gradients were estimated with the modified Bernoulli equation. The continuity equation was used to calculate the AVA. ${ }^{12}$ Severity of AS was categorised based on current recommendations. ${ }^{13}$

\section{Follow-up}

Patients were followed-up for the occurrence of allcause mortality. Survival data were complete for all subjects and collected from the departmental cardiology information system, which is linked to the respective national governmental death registry database. In addition, the occurrence and timing of aortic valve replacement (AVR) during follow-up were noted.

\section{Statistical analysis}

Continuous variables are presented as mean $\pm \mathrm{SD}$ and compared using the Student's t-test. All categorical variables are presented as percentages and compared using $\chi^{2}$ analysis or the Fisher exact test. Linear regression analysis was performed to assess the clinical and echocardiographic correlates of discordant grading moderate AS. The OR and 95\% CIs were calculated. Variables with a significant $\mathrm{p}$ value in the univariate analysis $(\mathrm{p}<0.05)$ were included in the multivariate analysis. Cumulative event rates for all-cause mortality were calculated using the Kaplan-Meier method and log-rank tests were used for comparisons between groups. The Cox proportional hazards models were used to estimate HRs and 95\% CI for the independent predictors of allcause mortality. Given that LVEF $<50 \%$ can account as a confounding factor in patients with discordant grading, subjects were categorised according to discordant/concordant grading and LVEF for purposes of survival analysis: (1) discordant moderate AS with LVEF $<50 \%$, (2) discordant moderate AS with LVEF $\geq 50 \%$, (3) concordant moderate AS and LVEF $<50 \%$ and (4) concordant moderate AS and LVEF $\geq 50 \%$. 


\begin{tabular}{|l|c|r|}
\hline & Aortic valve area & Mean gradient \\
\hline Concordant grading & $1.0-1.5 \mathrm{~cm}^{2}$ & $\geq 20 \mathrm{mmHg}$ \\
\hline Discordant grading & $1.0-1.5 \mathrm{~cm}^{2}$ & $<20 \mathrm{mmHg}$ \\
\hline
\end{tabular}

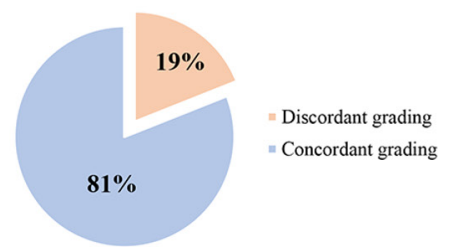

Figure 1 Classification of patients with moderate aortic stenosis according to concordance of mean gradient with aortic valve area. Discordant grading, moderate aortic stenosis has a considerable prevalence (19\%).

The variables included in the univariate Cox regression analysis were those that were significantly different between patients with concordant versus discordant grading. In the multivariate Cox regression analysis, a forward stepwise approach was used. A two-sided $p$ value $<0.05$ was considered as significant. All statistical analyses were performed using SPSS for Windows V.23 (SPSS, Armonk, New York: IBM).

\section{RESULTS}

\section{Study population}

Of 790 patients (mean age $71 \pm 12$ years, $52 \%$ men) diagnosed with moderate AS (defined by an AVA between $1.0 \mathrm{~cm}^{2}$ and $1.5 \mathrm{~cm}^{2}$ ) between 30 October 2001 and 5 June 2018, $150(19.0 \%)$ had discordant moderate AS (MG $<20 \mathrm{~mm} \mathrm{Hg}$ ) (figure 1). Tables 1 and 2 summarise the clinical and echocardiographic characteristics of the patients. Patients with discordant-grading moderate AS were significantly older, had higher prevalence of previous myocardial infarction and LV hypertrophy, higher LV filling pressure, larger LV end-diastolic and end-systolic volume index, lower LVEF and stroke volume index (SVi) as compared with patients with concordant grading moderate AS (figure 2). Table 3 summarises the clinical and echocardiographic correlates of discordant-grading moderate AS.

\section{Outcomes}

During a median follow-up of 4.9 (IQR 3.0-8.2) years, $40(26.7 \%)$ patients in the discordant grading group underwent aortic valve replacement (surgical or transcatheter) compared with $282(44.1 \%)$ patients in the concordant grading group and $90(60.0 \%)$ patients died in the discordant grading group compared with $276(43.1 \%)$ in the concordant grading group.

When assessing the all-cause mortality rates according to concordant/discordant grading and LVEF $<50 \%$ versus $\geq 50 \%$, the estimated death rates at 1,2 and 5 -year follow-up were, respectively, $13 \%, 31.2 \%$ and $75.3 \%$ for discordant-grading moderate AS with LVEF

\begin{tabular}{|c|c|c|c|}
\hline Variable & Discordant moderate AS ( $\mathrm{N}=150)$ & Concordant moderate AS ( $N=640)$ & $P$ value \\
\hline Age (years) & $74 \pm 10$ & $70 \pm 12$ & $<0.001$ \\
\hline Male gender, $\mathrm{n}(\%)$ & $88(58.7 \%)$ & $322(50.3 \%)$ & 0.065 \\
\hline Body mass index $\left(\mathrm{kg} / \mathrm{m}^{2}\right)$ & $25.0 \pm 4.6$ & $25.7 \pm 6.6$ & 0.213 \\
\hline Body surface area $\left(\mathrm{m}^{2}\right)$ & $1.70 \pm 0.25$ & $1.69 \pm 0.23$ & 0.510 \\
\hline Hypertension, n (\%) & $116(77.3 \%)$ & $493(77.2 \%)$ & 0.962 \\
\hline Diabetes mellitus, n (\%) & $59(39.3 \%)$ & $201(31.4 \%)$ & 0.063 \\
\hline Dyslipidaemia, $\mathrm{n}(\%)$ & $112(74.7 \%)$ & $486(76.1 \%)$ & 0.721 \\
\hline Coronary artery disease, $\mathrm{n}(\%)$ & $104(69.3 \%)$ & $278(43.4 \%)$ & $<0.001$ \\
\hline Previous myocardial infarction, $\mathrm{n}(\%)$ & $45(30.0 \%)$ & $78(12.2 \%)$ & $<0.001$ \\
\hline $\mathrm{eGFR}<60 \mathrm{~mL} / \mathrm{min} / 1.73 \mathrm{~m}^{2}, \mathrm{n}(\%)$ & $81(54.0 \%)$ & $257(41.1 \%)$ & 0.004 \\
\hline Chronic lung disease, $n$ (\%) & $16(10.7 \%)$ & $38(5.9 \%)$ & 0.039 \\
\hline Atrial fibrillation, $\mathrm{n}(\%)$ & $53(35.3 \%)$ & $160(25.0 \%)$ & 0.010 \\
\hline Beta blocker, n (\%) & $82(55.0 \%)$ & $317(49.5 \%)$ & 0.226 \\
\hline ACE inhibitor/ARB, n (\%) & $86(58.1 \%)$ & $310(48.4 \%)$ & 0.034 \\
\hline Calcium channel blockers, n (\%) & $49(32.9 \%)$ & $269(42 \%)$ & 0.040 \\
\hline Diuretics, n (\%) & $68(45.6 \%)$ & $207(32.3 \%)$ & 0.002 \\
\hline Statins, n (\%) & $123(82.6 \%)$ & $461(72.0 \%)$ & 0.008 \\
\hline
\end{tabular}

ACE, angiotensin converting enzyme; ARB, angiotensin-receptor blocker; AS, aortic stenosis; eGFR, estimated glomerular filtration rate. 


\begin{tabular}{lllr}
\hline Table 2 & Echocardiographic characteristics at baseline & & \\
\hline Variable & Discordant moderate AS (N=150) & Concordant moderate AS (N=640) & P value \\
\hline AVA $\left(\mathrm{cm}^{2}\right)$ & $1.23 \pm 0.16$ & $1.18 \pm 0.15$ & $<0.001$ \\
\hline Mean gradient $(\mathrm{mmHg})$ & $15.7 \pm 3.1$ & $28.5 \pm 6.4$ & $<0.001$ \\
\hline LV mass index $\left(\mathrm{g} / \mathrm{m}^{2}\right)$ & $125 \pm 35$ & $120 \pm 36$ & 0.093 \\
\hline LV end-diastolic diameter $(\mathrm{mm})$ & $52 \pm 7$ & $48 \pm 7$ & $<0.001$ \\
\hline LV end-diastolic volume $(\mathrm{mL})$ & $115 \pm 44$ & $105 \pm 37$ & 0.012 \\
\hline LV end-systolic volume $(\mathrm{mL})$ & $62 \pm 39$ & $42 \pm 25$ & $<0.001$ \\
\hline LV end-diastolic volume index $\left(\mathrm{mL}^{\prime} \mathrm{m}^{2}\right)$ & $70 \pm 29$ & $61 \pm 21$ & 0.003 \\
\hline LV end-systolic volume index $\left(\mathrm{mL} / \mathrm{m}^{2}\right)$ & $37 \pm 24$ & $25 \pm 15$ & $<0.001$ \\
\hline LV ejection fraction $(\%)$ & $49 \pm 16$ & $61 \pm 10$ & $<0.001$ \\
\hline Stroke volume index $\left(\mathrm{mL} / \mathrm{m}^{2}\right)$ & $42 \pm 11$ & $55 \pm 11$ & $<0.001$ \\
\hline Stroke volume index $<35 \mathrm{~mL} / \mathrm{m}^{2}$ & $38(26.6 \%)$ & $9(1.5 \%)$ & $<0.001$ \\
\hline LAVI (mL/m²) & $42.3 \pm 17.9$ & $41.2 \pm 25.5$ & 0.661 \\
\hline E/e' septal ratio & $21 \pm 10$ & $17 \pm 8$ & 0.002 \\
\hline LV hypertrophy, $\mathrm{n}(\%)$ & $387(61.4 \%)$ & 0.040 \\
\hline LVEF $<50 \%, \mathrm{n}(\%)$ & $58(9.1 \%)$ & $<0.001$ \\
\hline Moderate/severe MR, $\mathrm{n}(\%)$ & $42(6.6 \%)$ & 0.001 \\
\hline Moderate/severe TR, $\mathrm{n}(\%)$ & $105(70.5 \%)$ & $51(8.0 \%)$ & 0.186 \\
\hline TAPSE $<1.7 \mathrm{~cm}, \mathrm{n}(\%)$ & $22(14.7 \%)$ & $31(5.0 \%)$ & $<0.001$
\end{tabular}

AVA, aortic valve area; LAVI, left atrial volume index; LV, left ventricle; LVEF, left ventricle ejection fraction; MR, mitral regurgitation; TAPSE, tricuspid annular plane systolic excursion; TR, tricuspid regurgitation.

$<50 \%, 8 \%, 17.4 \%$ and $42.4 \%$ for discordant-grading moderate AS with LVEF $\geq 50 \%, 12.1 \%, 32.8 \%$ and $53.8 \%$ for concordant-grading moderate AS with LVEF $<50 \%$, and $3.8 \%, 10.3 \%$ and $31.6 \%$ for concordant-grading moderate AS with LVEF $\geq 50 \%$ (figure 3 ).

Table 4 outlines the univariate associates of all-cause mortality in the entire population. Older age, previous myocardial infarction, impaired renal function (estimated glomerular filtration rate $<60 \mathrm{~mL} / \mathrm{min} / 1.73 \mathrm{~m}^{2}$ ), LV hypertrophy, LV end-diastolic volume index, significant (moderate or severe) mitral regurgitation, SVi, TAPSE $<1.7 \mathrm{~cm}$ and concordant/discordant grading combined with LVEF were independently associated with mortality. On multivariate analysis, age, renal dysfunction, LV hypertrophy, discordant-grading moderate AS with LVEF $<50 \%$ (HR 2.78 (2.00-3.87), $\mathrm{p}<0.001)$ and concordant grading moderate AS with LVEF $<50 \%$ (HR 1.58 (1.06-2.36), $\mathrm{p}=0.025$ ) were independently associated with all-cause mortality.

\section{DISCUSSION}

The main findings of the present study are the relatively high frequency of patients with low gradient (MG

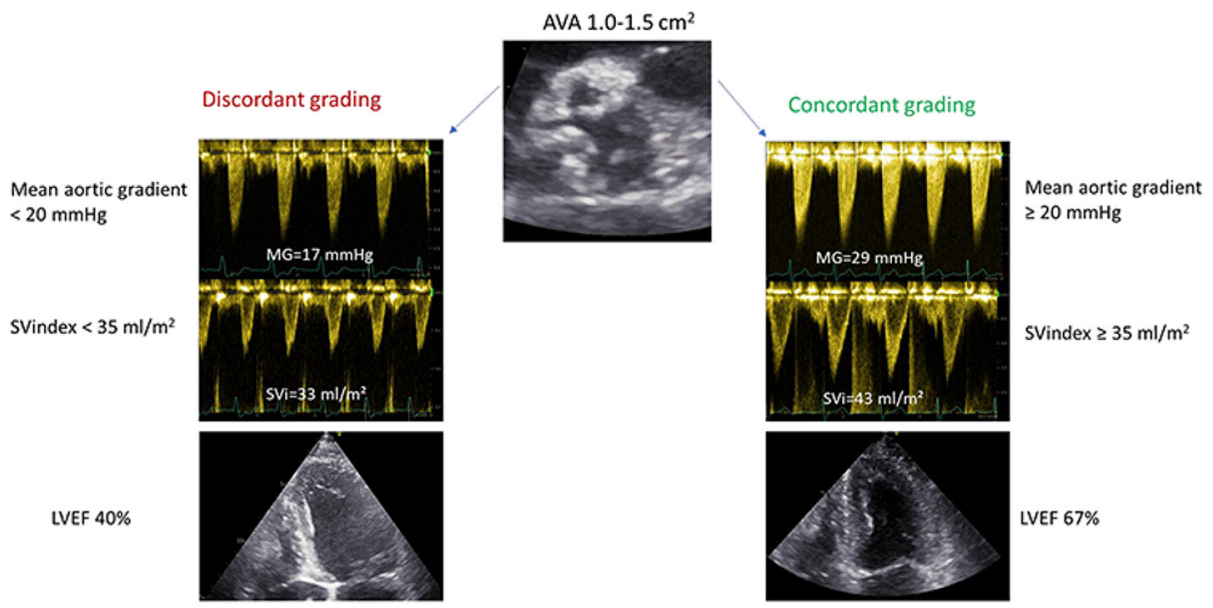

Figure 2 Echocardiographic differences between patients with discordant vs concordant grading. Patients with discordant grading, moderate AS significantly lower SVi and LVEF values compared with patients with concordant grading moderate AS. AS, aortic stenosis; AVA, aortic valve area; LVEF, left ventricular ejection fraction; SVi, stroke volume index. 
Valvular heart disease

Table 3 Clinical and echocardiographic correlates of discordant grading moderate AS

\begin{tabular}{|c|c|c|c|c|}
\hline & Univariate analysis & & Multivariate analysi & \\
\hline & Odds ratio $(95 \% \mathrm{Cl})$ & $P$ value & Odds ratio $(95 \% \mathrm{Cl})$ & $\mathbf{P}$ value \\
\hline Age (per 1 year increase) & $1.04(1.02$ to 1.05$)$ & $<0.001$ & 1.03 (1.01 to 1.06$)$ & 0.006 \\
\hline Male & 1.40 (0.98 to 2.01) & 0.066 & & \\
\hline Coronary artery disease (yes/no) & 2.94 (2.01 to 4.31$)$ & $<0.001$ & 1.74 (1.04 to 2.89$)$ & 0.034 \\
\hline Previous Ml (yes/no) & 3.08 (2.02 to 4.70$)$ & $<0.001$ & 1.44 (0.82 to 2.53$)$ & 0.207 \\
\hline LVEDV (per unit increase) & $1.01(1.00$ to 1.01$)$ & 0.006 & 1.00 (0.99 to 1.00$)$ & 0.368 \\
\hline LVEF (per unit increase) & $0.93(0.92$ to 0.94$)$ & $<0.001$ & 0.96 (0.94 to 0.98$)$ & $<0.001$ \\
\hline LV mass index (per unit increase) & $1.00(1.00$ to 1.01$)$ & 0.094 & & \\
\hline Moderate/severe MR (yes/no) & 2.45 (1.41 to 4.24$)$ & 0.001 & 1.56 (0.71 to 3.40$)$ & 0.265 \\
\hline Stroke volume index (per unit increase) & $0.88(0.86$ to 0.90$)$ & $<0.001$ & 0.90 (0.88 to 0.92$)$ & $<0.001$ \\
\hline Atrial fibrillation (yes/no) & 1.64 (1.12 to 2.40$)$ & 0.011 & $1.20(0.73$ to 1.98$)$ & 0.472 \\
\hline
\end{tabular}

AS, aortic stenosis; LV, left ventricular; LVEDV, left ventricular end-diastolic volume; LVEF, left ventricular ejection fraction; MI, myocardial infarction; MR, mitral regurgitation.

$<20 \mathrm{~mm} \mathrm{Hg}$ ), but AVA between $1.0 \mathrm{~cm}^{2}$ and $1.5 \mathrm{~cm}^{2}$ (discordant grading) among patients with moderate AS and its association with poor prognosis, particularly among patients with $\mathrm{LVEF}<50 \%$.

\section{Discordant grading in moderate AS}

Discordant grading in moderate AS was detected in $150(19.0 \%)$ patients in the present population, which is slightly lower than the reported prevalence of discordant grading in patients with severe AS (up to $30 \%) .{ }^{14}$ The most common findings that explain discordant grading in patients with AS are low flow status (low SVi), low LVEF, inaccurate measurement of LVOT cross-sectional area and misalignment of the aortic jet with the ultrasound beam. ${ }^{14}$ Patients with discordant grading moderate AS showed significantly lower SVi and LVEF values compared with patients with concordant-grading moderate AS. Furthermore,
AVA was significantly larger in the discordant-grading moderate AS, which would be expected since the classification is based on MG values, given that larger AVA yields a lower MG value. Conditions associated with low flow status such as coronary artery disease, larger LV volumes, moderate or severe mitral regurgitation and lower TAPSE were also more prevalent in the group with discordant grading. ${ }^{15} 16$ Although LVOT crosssectional area is measured as accurate as possible, the limitations of two-dimensional transthoracic echocardiography are well known, and correcting for body surface area should be performed, particularly in children, adolescents and women. ${ }^{11}$ Previous studies have reported on the frequency of discordant grading in patients with moderate AS. ${ }^{16-18}$

Tan and colleagues analysed flow and gradient patterns in patients with mild and moderate AS and
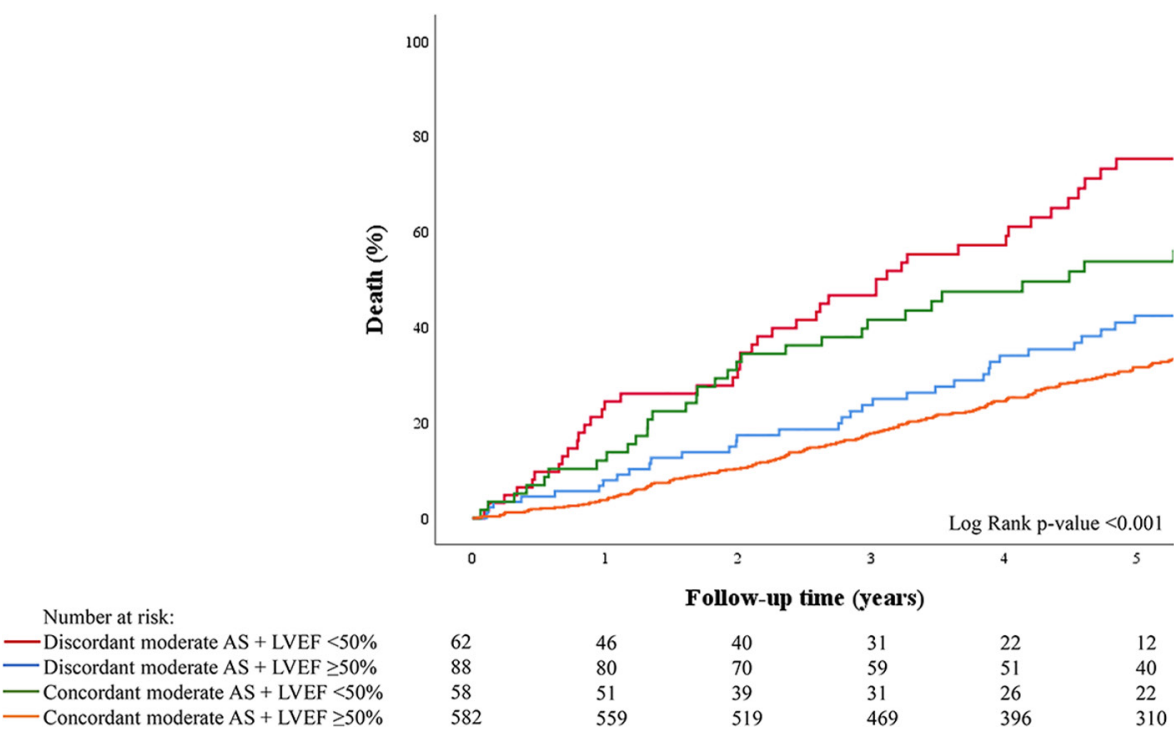

Figure 3 Kaplan-Meier estimates of all-cause mortality according to gradient concordance and LVEF. LVEF, left ventricular ejection fraction. 
Table 4 Uni- and multivariate Cox proportional hazard analyses for the identification of independent correlates of all-cause mortality

\begin{tabular}{|c|c|c|c|c|}
\hline & Univariate analysis & & Multivariate analysis & \\
\hline & Hazard ratio $(95 \% \mathrm{Cl})$ & $P$ value & Hazard ratio $(95 \% \mathrm{Cl})$ & $P$ value \\
\hline Age (per 1 year increase) & 1.05 (1.03 to 1.06$)$ & $<0.001$ & 1.04 (1.02 to 1.05$)$ & $<0.001$ \\
\hline Male & $1.00(0.81$ to 1.22$)$ & 0.973 & & \\
\hline Previous Ml (yes/no) & 1.72 (1.33 to 2.22$)$ & $<0.001$ & & \\
\hline $\mathrm{eGFR}<60 \mathrm{~mL} / \mathrm{min} / 1.73 \mathrm{~m}^{2}$ (yes/no) & 2.55 (2.07 to 3.15$)$ & $<0.001$ & 2.15 (1.71 to 2.70$)$ & $<0.001$ \\
\hline LV hypertrophy (yes/no) & 1.75 (1.39 to 2.20$)$ & $<0.001$ & 1.67 (1.30 to 2.15$)$ & $<0.001$ \\
\hline LVEDV index (per $1 \mathrm{~mL} / \mathrm{m}^{2}$ increase) & $1.007(1.003$ to 1.011$)$ & 0.001 & & \\
\hline Moderate/severe MR (yes/no) & 1.50 (1.05 to 2.13$)$ & 0.025 & & \\
\hline Stroke volume index (per unit increase) & 0.986 (0.977 to 0.996$)$ & 0.005 & & \\
\hline TAPSE $<1.7 \mathrm{~cm}$ (yes/no) & 1.71 (1.18 to 2.47$)$ & 0.004 & & \\
\hline Type of moderate AS & & & & \\
\hline Concordant moderate $\mathrm{AS}+\mathrm{LVEF} \geq 50 \%$ & Reference & $\ldots$ & Reference & $\ldots$ \\
\hline Discordant moderate AS +LVEF <50\% & 3.11 (2.26 to 4.27$)$ & $<0.001$ & 2.78 (2.00 to 3.87$)$ & $<0.001$ \\
\hline Discordant moderate AS + LVEF $\geq 50 \%$ & 1.46 (1.06 to 2.01$)$ & 0.022 & $1.01(0.78$ to 1.56$)$ & 0.595 \\
\hline Concordant moderate AS +LVEF $<50 \%$ & $2.12(1.49$ to 3.01$)$ & $<0.001$ & 1.58 (1.06 to 2.36$)$ & 0.025 \\
\hline
\end{tabular}

AS, aortic stenosis; eGFR, estimated glomerular filtration rate; LV, left ventricular; LVEDV, left ventricular end-diastolic volume; MI, myocardial infarction; MR, mitral regurgitation; TAPSE, tricuspid annular plane systolic excursion.

preserved LVEF and demonstrated a $70.3 \%$ prevalence of discordant grading in the moderate AS group. ${ }^{17}$ van Gils and colleagues analysed patients with moderate AS and reduced LVEF and reported a high rate of discordance MG $(81 \%)$ versus peak velocity $(84 \%){ }^{18}$ The demographic and clinical characteristics of the patients included in the later study are similar to those of the present population with high prevalence of comorbidities (hypertension, hyperlipidaemia and coronary artery disease). However, the prespecified criterium of reduced LVEF may explain the higher prevalence of discordant grading in that report as compared with the current results.

\section{Prognostic relevance of discordant grading in moderate AS}

In the present study, patients with discordant grading had worse prognosis as compared with patients with concordant grading. Few studies have evaluated the association between discordant grading and prognosis in patients with moderate AS. ${ }^{17} 18$ Delesalle and colleagues observed that among 508 patients with moderate AS and preserved LVEF, patients who were not referred for aortic valve replacement during follow-up had significantly lower MG as compared with patients who were operated $(23 \pm 8 \mathrm{~mm} \mathrm{Hg}$ vs $29 \pm 11 \mathrm{~mm} \mathrm{Hg}, \mathrm{p}<0.001) .{ }^{19}$ This suggests that among patients with moderate AS, there were patients with a low gradient that could mask the severity of the disease during follow-up (underdiagnosing severe AS), preventing the treating physician to refer these patients for intervention. In addition, van Gils and colleagues showed that patients with moderate AS and reduced LVEF ( $81 \%$ of them with a MG $<20 \mathrm{~mm} \mathrm{Hg}$ ) had a cumulative incidence of the composite of death, aortic valve replacement or heart failure hospitalisation of $61 \%$ at 4 years of follow-up. ${ }^{18}$ Although stroke volume is associated with poor prognosis in patients with severe $\mathrm{AS},{ }^{20}$ recent studies including patients with moderate AS do not confirm that low-flow status is an independent prognostic marker, ${ }^{21} 22$ and this is consistent in the present analysis.

Similarly, to the current study population, patients with calcific moderate AS have high prevalence of comorbidities $^{23}$ and increased incidence of cardiovascular events ${ }^{24}$ compared with the general population. In the present study, comorbidities such as prior myocardial and impaired renal function were associated with increased mortality. These comorbidities may lead to symptoms that confound the symptoms of moderate AS. Patients in the discordant moderate AS group are usually misclassified by MG values as having mild AS and the symptoms may be attributed to the comorbidities. Since AS does not have a predictable progression pattern, ${ }^{9}$ patients may be misclassified as having nonsevere AS and remain unoperated for a long period of time leading also to increased mortality. It has been postulated that the increased afterload imposed by the calcific stenotic aortic valve onto the LV may have a considerable role in the development of symptoms and increased risk of mortality in patients with heart failure with moderate AS. ${ }^{1025}$ Whether these patients will benefit from early aortic valve intervention will be elucidated by the ongoing trial TAVR-UNLOAD. ${ }^{10}$ 


\section{Study limitations}

This is a retrospective study, and the patients were determined to have moderate AS according to AVA, which may have introduced a selection bias. Additional studies to determine AS severity were not performed given the lack of guideline recommendation on the use of multimodality imaging in patients with moderate AS. The AS severity classification, clinical follow-up and the decision to replace the aortic valve were the responsibility of the treating physicians reflecting centre-specific practice and varied according to guideline recommendations throughout the years. Since the study was performed in tertiary referral centres, patients presented with significant comorbidities, which may explain the high mortality rate in this population. Low-dose dobutamine stress echocardiography was not systematically performed, since current recommendations do not include this test in patients with moderate AS. The inclusion of patients with significant mitral regurgitation and LV systolic dysfunction may result in a more representative sample of the moderate AS population.

\section{CONCLUSIONS}

Discordant grading moderate AS is not uncommon and is characterised by a high prevalence of comorbidities. The combination of discordant grading with moderate AS with low LVEF is associated with high risk of mortality.

\section{Author affiliations \\ ${ }^{1}$ Department of Cardiology, Leiden University Medical Center, Leiden, The Netherlands \\ ${ }^{2}$ Department of Cardiology, National Heart Centre, Singapore \\ ${ }^{3}$ Department of Cardiology, Royal Perth Hospital, Perth, Western Australia, Australia ${ }^{4}$ Department of Cardiothoracic Surgery, National Heart Centre Singapore, Singapore ${ }^{5}$ Institut Universitaire de Cardiologie et de Pneumologie de Québec, Québec Heart and Lung Institute, Laval University, Quebec, Quebec, Canada \\ ${ }^{6}$ Department of Cardiology, Thoraxcenter, Erasmus University Medical Center, Rotterdam, The Netherlands \\ ${ }^{7}$ Gangston Cardiovascular Institute, Morristown Medical Center, Morristown, New Jersey, USA \\ ${ }^{8}$ Department of Cardiology, Columbia University Medical Center, New York, New York, USA}

\section{Twitter Philippe Pibarot @ppibarot}

Contributors SMP: conception and design of the study; collection, analysis and interpretation of data; drafting of the manuscript; final approval of the manuscript; MRA: conception and design of the study; collection, analysis and interpretation of data; drafting of the manuscript; final approval of the manuscript; KYS: conception and design of the study; drafting of the manuscript; final approval of the manuscript; PP: conception and design of the study; drafting of the manuscript; final approval of the manuscript; NMVM: conception and design of the study; drafting of the manuscript; final approval of the manuscript; ZPD: conception and design of the study; drafting of the manuscript; final approval of the manuscript; PG: conception and design of the study; drafting of the manuscript; final approval of the manuscript: MBL: conception and design of the study; drafting of the manuscript; final approval of the manuscript; SHE: conception and design of the study; collection, analysis and interpretation of data; drafting of the manuscript; final approval of the manuscript; NAM: conception and design of the study; collection, analysis and interpretation of data; drafting of the manuscript; final approval of the manuscript: VD: conception and design of the study; collection, analysis and interpretation of data; drafting of the manuscript; final approval of the manuscript; JJB: conception and design of the study; collection, analysis and interpretation of data; drafting of the manuscript; final approval of the manuscript.

Funding SMP received funding from European Society of Cardiology in form of an ESC Training Grant (the reference of application number is T-2018-17405).

Competing interests The Department of Cardiology, Heart Lung Center, Leiden University Medical Center has received grants from Biotronik, Medtronic, Boston Scientific, GE Healthcare, and Edwards Lifesciences. NAM has received speaker fees from Abbott and Philips Ultrasound. ZPD has received speaker fees from Philips. PP has received grant support from Edwards Lifesciences and Medtronic. SHE has received speaker fees from Edwards Lifesciences and Abbott Vascular. VD has received speaker fees from Abbott Vascular. JJB has received speaker fees from Abbott Vascular and Boehringer Ingelheim. PG reports speaker fee from Edwards Lifesciences, Cordis, Medtronic; Consultant: Abiomed, Boston Scientific, Cardiovascular System Inc., Cordis, Edwards Lifesciences, Medtronic, Opsens, Soundbite Medical Solutions Inc., Pi-Cardia, Saranas, Siemens, Shockwave Medical Inc., SIG.NUM; Teleflex, 4C Medical, Shareholder: Soundbite Medical Solutions Inc., SIG.NUM, Pi-Cardia, Puzzle Medical. MBL is an early physician founder of Mitralign and has an equity relationship ( $<1 \%$ of the company).

Patient consent for publication Not required.

Ethics approval This retrospective analysis of clinically acquired data was approved by the respective institutional review boards of each participating centre and the need for patient written informed consent was waived due to the retrospective nature of the study.

Provenance and peer review Not commissioned; externally peer reviewed.

Data availability statement The data presented in the current manuscript are available upon reasonable request. The data are not in a repository and consist of deidentified participant data which can be available upon reasonable request at https://orcid.org/0000-0002-9841-2737.

Open access This is an open access article distributed in accordance with the Creative Commons Attribution Non Commercial (CC BY-NC 4.0) license, which permits others to distribute, remix, adapt, build upon this work non-commercially, and license their derivative works on different terms, provided the original work is properly cited, appropriate credit is given, any changes made indicated, and the use is non-commercial. See: http://creativecommons.org/licenses/by-nc/4.0/.

\section{ORCID iDs}

Philippe Pibarot http://orcid.org/0000-0002-3607-279X

Victoria Delgado http://orcid.org/0000-0002-9841-2737

\section{REFERENCES}

1 Minners J, Allgeier M, Gohlke-Baerwolf C, et al. Inconsistent grading of aortic valve stenosis by current guidelines: haemodynamic studies in patients with apparently normal left ventricular function. Heart 2010;96:1463-8.

2 Dumesnil JG, Pibarot P, Carabello B. Paradoxical low flow and/or low gradient severe aortic stenosis despite preserved left ventricular ejection fraction: implications for diagnosis and treatment. Eur Heart J 2010;31:281-9.

3 Clavel M-A, Messika-Zeitoun D, Pibarot P, et al. The complex nature of discordant severe calcified aortic valve disease grading: new insights from combined Doppler echocardiographic and computed tomographic study. J Am Coll Cardiol 2013;62:2329-38.

4 Berthelot-Richer M, Pibarot P, Capoulade R, et al. Discordant grading of aortic stenosis severity: echocardiographic predictors of survival benefit associated with aortic valve replacement. JACC Cardiovasc Imaging 2016;9:797-805.

5 Eleid MF, Sorajja P, Michelena HI, et al. Flow-gradient patterns in severe aortic stenosis with preserved ejection fraction: clinical characteristics and predictors of survival. Circulation 2013;128:1781-9.

6 Le Ven F, Freeman M, Webb J, et al. Impact of low flow on the outcome of high-risk patients undergoing transcatheter aortic valve replacement. J Am Coll Cardiol 2013;62:782-8.

7 Mohty D, Magne J, Deltreuil M, et al. Outcome and impact of surgery in paradoxical low-flow, low-gradient severe aortic stenosis and preserved left ventricular ejection fraction: a cardiac catheterization study. Circulation 2013;128:S235-42.

8 Samad Z, Vora AN, Dunning A, et al. Aortic valve surgery and survival in patients with moderate or severe aortic stenosis and left ventricular dysfunction. Eur Heart J 2016;37:2276-86.

9 Rosenhek R, Klaar U, Schemper M, et al. Mild and moderate aortic stenosis. natural history and risk stratification by echocardiography. Eur Heart J 2004;25:199-205. 
10 Spitzer E, Van Mieghem NM, Pibarot P, et al. Rationale and design of the Transcatheter Aortic Valve Replacement to UNload the Left ventricle in patients with Advanced heart failure (TAVR UNLOAD) trial. Am Heart J 2016;182:80-8.

11 Baumgartner $\mathrm{H}$, Hung J, Bermejo J, et al. Recommendations on the echocardiographic assessment of aortic valve stenosis: a focused update from the European Association of Cardiovascular Imaging and the American Society of Echocardiography. J Am Soc Echocardiogr 2017;30:372-92.

12 Prihadi EA, Vollema EM, Ng ACT, et al. Determinants and prognostic implications of left ventricular mechanical dispersion in aortic stenosis. Eur Heart J Cardiovasc Imaging 2019;20:740-8.

13 Lang RM, Badano LP, Mor-Avi V, et al. Recommendations for cardiac chamber quantification by echocardiography in adults: an update from the American Society of Echocardiography and the European Association of Cardiovascular Imaging. Eur Heart $J$ Cardiovasc Imaging 2015;16:233-71.

14 Delgado V, Clavel M-A, Hahn RT, et al. How do we reconcile echocardiography, computed tomography, and hybrid imaging in assessing discordant grading of aortic stenosis severity? JACC Cardiovasc Imaging 2019;12:267-82.

15 Minners J, Allgeier M, Gohlke-Baerwolf C, et al. Inconsistencies of echocardiographic criteria for the grading of aortic valve stenosis. Eur Heart J 2008;29:1043-8.

16 Leong DP, Pizzale S, Haroun MJ, et al. Factors associated with low flow in aortic valve stenosis. J Am Soc Echocardiogr 2016;29:158-65.

17 Tan Y-QB, Ngiam JN, Kong WKF, et al. Prevalence, clinical and echocardiographic characteristics of various flow and gradient patterns in mild or moderate aortic stenosis with normal left ventricular ejection fraction. Int J Cardiol 2016;221:1107-15.

18 van Gils L, Clavel M-A, Vollema EM, et al. Prognostic implications of moderate aortic stenosis in patients with left ventricular systolic dysfunction. J Am Coll Cardiol 2017;69:2383-92.

19 Delesalle G, Bohbot Y, Rusinaru D, et al. Characteristics and prognosis of patients with moderate aortic stenosis and preserved left ventricular ejection fraction. J Am Heart Assoc 2019;8:e011036.

20 Capoulade R, Le Ven F, Clavel M-A, et al. Echocardiographic predictors of outcomes in adults with aortic stenosis. Heart 2016;102:934-42.

21 Mann TD, Loewenstein I, Ben Assa E, et al. Natural history of moderate aortic stenosis with preserved and low ejection fraction. $J$ Am Soc Echocardiogr 2021. doi:10.1016/j.echo.2021.02.014. [Epub ahead of print: 27 Feb 2021].

22 Du Y, Gössl M, Garcia S, et al. Natural history observations in moderate aortic stenosis. BMC Cardiovasc Disord 2021;21:108.

23 Gharacholou SM, Karon BL, Shub C, et al. Aortic valve sclerosis and clinical outcomes: moving toward a definition. Am J Med 2011;124:103-10.

24 Otto CM, Lind BK, Kitzman DW, et al. Association of aortic-valve sclerosis with cardiovascular mortality and morbidity in the elderly. $N$ Engl J Med 1999;341:142-7.

25 Pibarot P, Messika-Zeitoun D, Ben-Yehuda O. Moderate aortic stenosis and heart failure with reduced ejection fraction: can imaging guide us to therapy? J Am Coll Cardiol Img 2019;12:172-84. doi:10.1016/j.jcmg.2018.10.021 